\title{
Disciplina de mercado en el sistema bancario centroamericano
}

\author{
Market discipline in the Central American banking system \\ Edgar Demetrio Tovar-García ${ }^{a, b}$ \\ a Universidad Panamericana, México \\ ${ }^{\mathrm{b}}$ Universidad de Monterrey, México
}

Recibido el 20 de noviembre de 2016; aceptado el 6 de julio de 2017

Disponible en Internet el 1 de noviembre de 2017

\section{Resumen}

Se verifica empíricamente la hipótesis de disciplina de mercado en el sistema bancario centroamericano. Se contrasta si los bancos más riesgosos (con peores fundamentales bancarios) pagan tasas de interés más altas y reciben menores cantidades de depósitos. Se utiliza el método general de momentos para modelos dinámicos con datos de panel (el estimador SYS GMM) y una muestra de 30 bancos de seis países centroamericanos durante el periodo 2008-2012. A diferencia de la mayor parte de la literatura empírica previa, particularmente para países desarrollados, en Centroamérica no se encontró evidencia de disciplina de mercado. Los resultados son robustos a varios indicadores de los fundamentales bancarios, al efecto de la demanda interna de capital del banco y a otros métodos econométricos. Estos hallazgos indican debilidades en la política de revelación de información bancaria.

(C) 2017 Universidad Nacional Autónoma de México, Facultad de Contaduría y Administración. Este es un artículo Open Access bajo la licencia CC BY-NC-ND (http://creativecommons.org/licenses/by-nc-nd/4.0/).

\section{Códigos JEL: E59; G21; G39}

Palabras clave: Disciplina de mercado; Mercado interno de capital; Mercado de depósitos; Riesgo bancario; Centroamérica

\footnotetext{
Abstract

This article empirically tests the market discipline hypothesis in the Central American banking system. Whether the riskier banks (with the worst bank fundamentals) pay higher interest rates and attract fewer amounts of deposits. We use dynamic panel data models and the generalized method of moments

Correo electrónico: demetrio.tovar@gmail.com

La revisión por pares es responsabilidad de la Universidad Nacional Autónoma de México. 
(SYS GMM) estimator, and a sample of 30 banks from six Central American countries over the years 2008-2012. In contrast to most of the previous empirical literature, particularly in developed countries, in Central America we did not find evidence for market discipline. The results are robust to different indicators of the bank fundamentals, to the effect of the internal demand for funding by banks, and to other econometric methods. These findings indicate weakness in the disclosure policy of banking information.

(C) 2017 Universidad Nacional Autónoma de México, Facultad de Contaduría y Administración. This is an open access article under the CC BY-NC-ND license (http://creativecommons.org/licenses/by-nc-nd/4.0/).

JEL classification: E59; G21; G39

Keywords: Market discipline; Internal capital market; Deposit market; Bank risk; Central America

\section{Introducción}

Tras la crisis financiera global con origen en Estados Unidos en 2007-2008, el Comité de Supervisión Bancaria de Basilea reevaluó sus recomendaciones fundamentales en el año 2010, dando lugar a los acuerdos de Basilea III. Se reformularon criterios para la estabilidad bancaria, pero continuando con los tres pilares enunciados en Basilea II: requerimientos mínimos de capital, supervisión bancaria y disciplina de mercado (Ayadi, 2013; Martínez Castillo, 2007).

El tercer pilar propone una política de revelación de la información bancaria para disminuir los problemas de riesgo moral y selección adversa. Dicha política, por un lado, resulta costosa e incómoda para los bancos, y por otro lado, beneficiosa para la sociedad, ya que puede favorecer la estabilidad del sistema bancario (Basel Committee on Banking Supervision, 2006, 2011, 2014). Dicha información sería utilizada por los agentes económicos, quienes tomarían mejores decisiones y, a través de mecanismos de mercado, precios y cantidades, disciplinarían a los bancos que incurren en riesgos excesivos, a la vez que envían señales de mercado a los supervisores bancarios (Tovar-García, 2014).

Evidentemente, ni el mercado ni los supervisores fueron capaces de prever y prevenir las últimas crisis bancarias. No obstante, el tercer pilar es todavía clave en las recomendaciones de Basilea, que actualmente busca mejorar la comparabilidad y la consistencia de la información a revelar (Basel Committee on Banking Supervision, 2014). En este contexto, la presente investigación verifica la presencia de disciplina de mercado en el sistema bancario centroamericano. Se contrasta la hipótesis de que los bancos más riesgosos pagan tasas de interés más altas y atraen una menor cantidad de depósitos.

La hipótesis de disciplina de mercado ha sido verificada positivamente sobre todo en países desarrollados (Berger y Turk-Ariss, 2014); también hay evidencia de su presencia en países exsocialistas (Hasan, Jackowicz, Kowalewski y Kozłowski, 2013), en China (Wu y Bowe, 2012) y en América Latina (Martinez-Peria y Schmukler, 2001). En América Central la hipótesis ha sido contrastada únicamente en el caso costarricense, con evidencia débil a su favor (Mayorga Martínez y Muñoz Salas, 2002). No obstante, hallazgos recientes sugieren que las pruebas empíricas previas están sesgadas, ya que no consideran la demanda interna de capital por parte de los bancos (Ben-David, Palvia y Spatt, 2016; Tovar-García, 2014).

La presente investigación utiliza información contable (proveniente del balance general y del estado de resultados) de los años 2008 a 2012 de 30 bancos con presencia en Costa Rica, El Salvador, Guatemala, Honduras, Nicaragua y Panamá. El estudio contribuye a la literatura de tres formas. Primero, se reevalúa la hipótesis en un nuevo contexto, donde la mayoría de los países centroamericanos tienen seguros de depósito y bajo la influencia de la crisis financiera global. 
Segundo, se emplea el método general de momentos y un modelo dinámico para datos de panel, el estimador SYS GMM (Blundell y Bond, 1998), que hasta ahora ha sido poco utilizado para corroborar la presencia de disciplina de mercado pero cuyas propiedades controlando endogeneidad lo hacen más atractivo que los típicos métodos con efectos fijos o flexibles. Tercero, se controla el efecto del mercado interno de capital, largamente ignorado en la literatura previa.

A diferencia de la mayor parte de los resultados previos, en particular en países desarrollados, los resultados aquí encontrados sugieren la ausencia de disciplina ejercida por los depositantes. Estos hallazgos son robustos a distintas especificaciones y métodos. La principal implicación para la política económica es la necesidad de restaurar la disciplina de mercado. Los depositantes deben entender que ellos también tienen un papel como vigilantes del riesgo bancario y los encargados de la política económica deben seguir las recomendaciones del Comité de Basilea sobre la política de revelación de información bancaria; en caso contrario, el riesgo de crisis bancaria se eleva.

El resto del artículo procede de la siguiente manera. La siguiente sección revisa brevemente la literatura. Posteriormente se presentan las principales variables y los correspondientes estadísticos descriptivos. Luego se desarrolla el modelo econométrico, se presentan las estimaciones y se discuten los resultados. Finalmente, se cierra con una sección de conclusiones.

\section{Breve revisión de la literatura}

La disciplina de mercado en el sistema bancario consiste en la reacción de los agentes económicos ante la toma de riesgo de los bancos. Desde el lado de los pasivos bancarios se asume que los depositantes y otros acreedores bancarios encuentran la toma excesiva de riesgo por parte del banco como una situación que eleva sus costos, es decir, eleva el riesgo de que el banco no pueda hacer frente a sus obligaciones ${ }^{1}$. Por consiguiente, los depositantes (en el caso de esta investigación) solicitarán una mayor tasa de interés por sus depósitos y/o pueden retirar sus recursos como respuesta a la toma excesiva de riesgo de su banco (Demirgüç-Kunt y Huizinga, 2004) ${ }^{2}$.

Lo anterior se entiende fácilmente con el bien conocido modelo de oferta y demanda. En el mercado de depósitos, la curva de oferta (depositantes) se desplazará hacia la izquierda como respuesta a la toma excesiva de riesgo por parte del banco, arribando a un nuevo punto de equilibrio en el que se tendrá una tasa de interés más alta y una menor cantidad.

Así, las pruebas tradicionales de disciplina de mercado verifican dos hipótesis:

H1. Los bancos más riesgosos pagan tasas de interés más altas (mecanismo de disciplina de mercado vía precio).

H2. Los bancos más riesgosos atraen una menor cantidad de depósitos (mecanismo de disciplina de mercado vía cantidad).

\footnotetext{
${ }^{1}$ La literatura sugiere que los tenedores de deuda subordinada son los más interesados en monitorear el riesgo bancario (Tovar-García, 2015). Asimismo, los bancos vigilan la toma de riesgo de sus colegas en el mercado interbancario (TovarGarcía, 2016a, 2016b). Además, la disciplina del mercado también puede provenir del lado de los activos bancarios, es decir, los prestatarios también tienen interés en monitorear el riesgo bancario y disciplinan a los bancos porque prefieren solicitar préstamos de bancos bien capitalizados y con calidad en sus activos, por motivos de refinanciamiento y señalización (Tovar-García, 2012; Tovar-García y Kozubekova, 2016). Estas hipótesis sobrepasan los límites de esta investigación, y por ello no se verifican para el caso centroamericano.

${ }^{2}$ Los depositantes también pueden modificar los plazos de sus depósitos, prefiriendo los de corto plazo como respuesta a la toma excesiva de riesgo bancario (Goday, Gruss y Ponce, 2005; Tovar-García, 2014). Por falta de información, aquí no se verificará este tercer mecanismo.
} 
Las pruebas empíricas consisten en estimaciones de la curva de oferta de depósitos con base en la forma reducida, ya que la carencia de datos hace imposible estimar simultáneamente las curvas de oferta y demanda (Park, 1995; Tovar-García, 2014), donde las variables dependientes son la tasa de interés y la tasa de crecimiento de los depósitos, y la principal variable independiente es el riesgo bancario.

La disciplina de mercado asume que los depositantes son capaces de entender y monitorear los riesgos bancarios; si esto no es así, es por falta de transparencia e información bancaria. Por ello, la política de revelación de información bancaria propuesta por Basilea es importante. Además, se asume que el banco también reaccionará, disminuyendo sus riesgos, para evitar pagar tasas de interés más altas y atraer fondos. Es en esta última fase que la disciplina de mercado se concreta (Flannery, 2001).

Las dos hipótesis mencionadas arriba han sido ampliamente corroboradas alrededor del mundo. Principalmente, las pruebas empíricas soportan la presencia de disciplina de mercado en países desarrollados (Berger y Turk-Ariss, 2014). En cambio, en los países en vías de desarrollo la evidencia es contradictoria o mixta.

En los países en transición, como Rusia, la evidencia empírica sugiere que los depositantes monitorean la toma de riesgo de sus bancos y modifican primordialmente las cantidades depositadas. Los depositantes rusos responden principalmente a indicadores de capitalización bancaria, liquidez y calidad crediticia para decidir el volumen de sus depósitos. No obstante, en Rusia, el mecanismo de disciplina vía precio (vía la tasa de interés sobre los depósitos) débilmente responde al riesgo bancario (Karas, Pyle y Schoors, 2010). En otros países de Europa Central y del Este los hallazgos son similares, es decir, la evidencia a favor de la hipótesis de disciplina de mercado se encuentra esencialmente a través del mecanismo de disciplina de mercado vía cantidad (Hasan et al., 2013). Por lo tanto, se puede decir que la disciplina de mercado existe en estos países. Quizás, a diferencia de otras naciones en vías de desarrollo, en los países en transición los depositantes aún desconfían de las capacidades del gobierno para llevar a cabo rescates bancarios. Así lo sugieren la crisis bancaria y financiera de Rusia en 1995 y 1998 (Karas et al., 2010). En Kirguistán, por ejemplo, en 2010 fue imposible rescatar al banco más grande del país, por lo que los agentes económicos entienden que hay bancos demasiado grandes para ser rescatados y vale la pena monitorear la toma de riesgo bancario desconfiando de la supervisión gubernamental (Tovar-García y Kozubekova, 2016).

En las naciones emergentes, como China, los resultados empíricos también sugieren que la tasa de crecimiento de los depósitos reacciona a la toma de riesgo bancario. Más aún, los bancos con mayores niveles de transparencia e información bancaria son capaces de atraer más depositantes (Wu y Bowe, 2012). Por el contrario, en India, otro de los grandes países emergentes, no se encuentra ninguna evidencia a favor de la existencia de disciplina de mercado, lo que hace cuestionarse hasta qué punto puede el marcado cumplir con tareas de vigilancia bancaria, especialmente en naciones con mercados financieros poco desarrollados (Sarkar y Bhole, 2009).

En América Latina la evidencia también es mixta (véase Tovar-García, 2014, tabla 5, p. 24), y hallazgos recientes sugieren una disciplina de mercado débil en el caso mexicano (Tovar-García, 2014), en contraste con la evidencia positiva que encontraron Martinez-Peria y Schmukler (2001) en los años noventa y principalmente después de la crisis mexicana en 1994-1995.

Es interesante reconocer que los pequeños depositantes prestan poca atención a los indicadores de riesgo o fundamentales bancarios; ellos se guían por la mercadotecnia, a diferencia de los grandes depositantes, quienes poseen un gran porcentaje del total de depósitos, educación financiera y recursos para monitorear a sus bancos, tal como sugiere la evidencia colombiana (Márquez, 2011). 
Además, en los países en vías de desarrollo se suele argumentar que las pruebas de disciplina de mercado vía precio pueden estar sesgadas por información imperfecta que caracteriza a estos mercados y porque depende de la aversión al riesgo de los depositantes (Mayorga Martínez y Muñoz Salas, 2002; Park y Peristiani, 1998; Park, 1995; Tovar-García, 2014). En concordancia, en Uruguay se encuentra fuerte evidencia a favor de la hipótesis de disciplina de mercado a través del mecanismo basado en cantidad y débil a través del precio (Goday, Gruss y Ponce, 2005). En el caso venezolano se encuentra evidencia favorable solamente a través del mecanismo basado en cantidad (Muñoz, Cabeza y Guerra, 2013).

En Centroamérica, la hipótesis de disciplina de mercado ha sido contrastada solo para el caso costarricense. Mayorga Martínez y Muñoz Salas (2002) utilizan información mensual de 21 bancos en el periodo 1995-2001 para verificar la hipótesis de disciplina de mercado vía cantidad ${ }^{3}$. Con base en regresiones con efectos fijos, sus resultados sugieren una presencia débil de disciplina de mercado, donde los fundamentales bancarios (principales indicadores de riesgo bancario) e incluso las variables macroeconómicas no parecen influir el comportamiento de los depósitos.

Hallazgos recientes para Estados Unidos también rechazan la presencia de disciplina de mercado cuando las pruebas controlan el efecto de la demanda interna de capital del banco, sugiriendo que los resultados previos están sesgados (Ben-David et al., 2016). Las pruebas de disciplina de mercado asumen que la tasa de interés (y la cantidad de depósitos) son determinadas principalmente por la oferta. Sin embargo, es importante reconocer que una tasa de interés más alta puede resultar de cambios en la curva de demanda (por parte del banco), y este efecto puede ser controlado utilizando la tasa de crecimiento de los préstamos (Ben-David et al., 2016; Tovar-García, 2014).

Una tasa de interés por los depósitos más alta puede ser resultado del desplazamiento de la curva de oferta (como se explicó arriba), pero también puede ser resultado de un desplazamiento de la curva de demanda hacia la derecha. Si el banco enfrenta una mayor demanda de préstamos (incremento en la tasa de crecimiento de los préstamos), para hacer frente a ella puede pagar tasas de interés más altas para atraer más depósitos. Ben-David et al. (2016) encuentran que la capitalización bancaria (principal indicador de riesgo bancario) no determina la tasa de interés por depósitos, en contra de la hipótesis de disciplina de mercado. Por el contrario, la tasa de crecimiento de los préstamos es el principal determinante a favor de la hipótesis de la demanda interna de capital del banco.

\section{Datos y estadísticos descriptivos}

Los datos bancarios empleados en esta investigación provienen de la agencia Bankscope y la información macroeconómica fue obtenida del Banco Mundial (World Development Indicators [WDI]). Actualmente, en los países centroamericanos bajo estudio hay 159 bancos clasificados por Bankscope como comerciales: 19 en Costa Rica, 14 en El Salvador, 24 en Guatemala, 19 en Honduras, 7 en Nicaragua y 76 en Panamá. Sin embargo, muchos de los indicadores clave empleados en esta investigación no están disponibles para muchos de estos bancos y, tras una revisión y eliminación de valores extremos, la muestra aquí empleada incluye 30 bancos. Varios de estos bancos son considerados grandes en sus respectivos países, es decir, tienen un porcentaje alto de participación en los distintos mercados bancarios. Un ranking de los bancos puede verse en Ríos (2012).

\footnotetext{
${ }^{3}$ La carencia de datos les imposibilitó verificar la hipótesis vía precio.
} 
Tabla 1

Muestra de bancos centroamericanos

\begin{tabular}{lll}
\hline País & Número de bancos & Nombre de los bancos \\
\hline Costa Rica & 2 & Banco Nacional de Costa Rica; Banco Lafise SA \\
El Salvador & 5 & Banco Agrícola; Scotiabank El Salvador SA; Banco Davivienda Salvadoreño, \\
& & SA; Banco Citibank de El Salvador SA; Banco G\&T Continental El Salvador \\
Guatemala & 2 & Banco Industrial SA; Banco de Desarrollo Rural SA \\
Honduras & 1 & Banco Financiera Centroamericana (FICENSA) \\
Nicaragua & 1 & Banco Citibank de Nicaragua SA \\
Panamá & 19 & Banistmo SA; Bancolombia (Panamá) SA; Global Bank Corporation; Banesco \\
& & S.A.; Banco Bilbao Vizcaya Argentaria (Panamá) SA; Banco Internacional de \\
& Costa Rica; Credicorp Bank SA; GTC Bank Inc; Towerbank International Inc.; \\
& & Metrobank SA; Capital Bank Inc; Popular Bank Ltd Inc; BCT Bank \\
& International; MMG Bank Corporation; Banco Universal S.A.; Banco Lafise \\
& Panamá, S.A.; BAC Bank Inc; Banco Delta, S.A. (BMF); Banco Azteca \\
& (Panamá) SA \\
& & \\
Total & 30 &
\end{tabular}

Fuente: Elaboración propia.

Diecinueve bancos (más del 60\% de la muestra) operan en Panamá. No se tiene información de los bancos panameños mejor clasificados, pero se tiene información de varios bancos panameños posicionados en la región entre el número 13 (Global Bank Corporation) y el número 128 (Banco Azteca-Panamá) de 136 bancos clasificados con respecto a su tamaño en 2011. Se tienen datos de cinco de los 14 bancos operando en El Salvador, y tres de ellos son los más grandes del país. En Nicaragua solo se tiene información completa de Citibank (antes Banco Uno), clasificado en la región como el banco 91 a finales de 2011; en esa fecha era el quinto banco más grande del país, pero con un modesto $5.8 \%$ de la cartera de crédito. En Honduras se tiene información únicamente de Banco Financiera Centroamericana (FICENSA), clasificado en la región como el banco 67, pero es el noveno banco del país. En Costa Rica se tiene información de uno de los principales bancos estatales, Banco Nacional de Costa Rica, que es el banco más grande del país, con el $26.5 \%$ de la cartera de crédito y posicionado en la región como el cuarto banco más grande. El segundo banco costarricense en la muestra es un banco extranjero de propiedad nicaragüense, LAFISE, el décimo banco del país y clasificado en la región como el número 73. Finalmente, en Guatemala se tiene información de Banco Industrial, clasificado en la región como el quinto banco más grande y posicionado como el primero en el país, con el $26 \%$ de la cartera de crédito. El segundo banco guatemalteco en la muestra es un banco estatal, Banco de Desarrollo Rural ${ }^{4}$, clasificado en la región como el undécimo banco más grande y el cuarto banco más grande del país (tabla 1 y Ríos, 2012) . $^{5}$.

Se analizan los años 2008 a 2012 porque la información bancaria es mucho más completa para dicho periodo y porque incluye los efectos de la crisis financiera global, que si bien no produjo tasas negativas de crecimiento en todos los países centroamericanos, sí redujo considerablemente

\footnotetext{
${ }^{4}$ Cabe señalar que en la muestra hay otros pocos bancos de propiedad estatal, pero no se espera que estos bancos puedan sesgar los resultados de la investigación. Además, interesa verificar la presencia de la disciplina de mercado para todo el sector bancario y no solo para la banca privada.

${ }^{5}$ La muestra es una limitación del trabajo y demerita la representatividad de la misma. No obstante, el análisis con datos de panel permite un análisis en el tiempo y en el espacio que favorece generalizar los resultados teniendo en cuenta las limitaciones señaladas.
} 
las tasas de crecimiento, principalmente en el año 2009. Para ese año las respectivas tasas son: $-3.1 \%$ en El Salvador, $-2.4 \%$ en Honduras, $-2.2 \%$ en Nicaragua, $-1.2 \%$ en Costa Rica, $0.5 \%$ en Guatemala y $3.8 \%$ en Panamá.

Existen dos posturas contrarias con respecto al efecto que las crisis bancarias y económicas tienen sobre la disciplina de mercado en el sector bancario. Martinez-Peria y Schmukler (2001) sostienen que estas favorecen la disciplina de mercado, principalmente después de la crisis, y representan un llamado a monitorear los riesgos bancarios por parte de los depositantes y otros acreedores bancarios. Por el contrario, hallazgos recientes sugieren que las crisis bancarias debilitan la disciplina de mercado (Cubillas, Fonseca y González, 2012). No tenemos datos suficientes para corroborar la hipótesis de trabajo en periodos de precrisis, crisis y poscrisis; no obstante, dado que las crisis global tuvo origen en Estados Unidos, con fuertes efectos en países europeos y sus sistemas bancarios, y con una gran cobertura mediática, se asume que los depositantes centroamericanos deberían estar mucho más alerta durante el periodo de estudio.

\section{Tasa de interés y crecimiento de los depósitos}

Dadas las limitaciones para obtener información directa sobre las tasas de interés pagadas por los bancos a los depositantes, aquí se utiliza una estrategia común en la literatura empírica. Se emplea una tasa de interés implícita calculada como la razón entre los gastos por intereses debido a depósitos y el total de depósitos (en adelante, tasa de interés por depósitos). Esta será la variable dependiente que permitirá verificar la hipótesis de disciplina de mercado con base en un mecanismo de precio. En la muestra, la tasa de interés por depósitos promedio es del $3.4 \%$ y su desviación estándar es del $2 \%$ (tabla 2).

En países en vías de desarrollo la disciplina de mercado con base en un mecanismo de precio puede estar sesgada por información imperfecta, como se mencionó arriba; por lo tanto, es relevante verificar el mecanismo basado en cantidad. Para ello se utiliza la tasa de crecimiento de los depósitos totales expresada en porcentaje, es decir, depósitos actuales entre depósitos del año previo. La tasa de crecimiento de los depósitos promedio es del 15.6\% y su desviación estándar, del $25.2 \%$ (tabla 2).

\section{Fundamentales bancarios: Sistema de clasificación CAMUL}

Para verificar la hipótesis de trabajo la principal variable independiente es riesgo bancario, lo cual puede ser aproximado utilizando las calificaciones otorgadas por diferentes agencias de calificación crediticia. Sin embargo, estas agencias otorgan calificaciones para un reducido número de bancos centroamericanos; por consiguiente, aquí se utiliza una estrategia común en la literatura empírica recurriendo a los fundamentales bancarios basados en el sistema de clasificación CAMUL (capitalización, calidad de activos, administración, utilidades y liquidez) ${ }^{6}$. Los estadísticos descriptivos de estas variables se resumen en la tabla 2.

El nivel de capitalización bancaria es aproximado con el ratio de adecuación del capital, es decir, capital básico (Tier 1) más capital complementario (Tier 2), que pondera el capital con respecto al riesgo en los activos, conforme a las reglas de Basilea.

La calidad de los activos es medida con la razón entre reservas para préstamos de dudosa recuperación y el total de préstamos dudosos (morosos). Entre mayor sea esta razón mejor, mayor

${ }^{6}$ CAMEL, conforme a las siglas en inglés (capital adequacy, asset quality, management quality, earnings and liquidity). 
Tabla 2

Estadísticos descriptivos

\begin{tabular}{|c|c|c|c|c|c|}
\hline Variable & Obs & Media & $\begin{array}{l}\text { Desviación } \\
\text { estándar }\end{array}$ & Mínimo & Máximo \\
\hline Activos totales $^{\mathrm{a}}$ & 150 & $2,012,610$ & $2,784,982$ & 24,380 & $1.38 \mathrm{E}+07$ \\
\hline Depósitos totales ${ }^{\mathrm{a}}$ & 150 & $1,454,333$ & $1,954,988$ & 10,219 & $1.06 \mathrm{E}+07$ \\
\hline Tasa de interés por depósitos & 150 & 3.4 & 2.0 & 0.4 & 19.9 \\
\hline Tasa de crecimiento de los depósitos & 149 & 15.6 & 25.2 & -31.7 & 173.0 \\
\hline Tasa de crecimiento de los préstamos & 148 & 13.9 & 24.1 & -36.6 & 152.9 \\
\hline Ratio de adecuación del capital & 150 & 18.1 & 11.3 & 8.4 & 83.8 \\
\hline $\begin{array}{l}\text { Reservas para préstamos dudosos/total } \\
\text { de préstamos dudosos }\end{array}$ & 127 & 172.5 & 140.1 & 36.9 & 883.1 \\
\hline Préstamos dudosos/total de préstamos & 127 & 2.2 & 2.5 & 0.1 & 21.5 \\
\hline ROA (ingreso neto/activos totales promedio) & 150 & 1.5 & 1.3 & -1.6 & 8.0 \\
\hline ROE (ingreso neto/acciones totales promedio) & 150 & 13.4 & 7.5 & -12.2 & 38.8 \\
\hline $\begin{array}{l}\text { Gastos no vinculados con intereses/total } \\
\text { de ingresos }\end{array}$ & 150 & 56.8 & 15.5 & 24.5 & 117.3 \\
\hline $\begin{array}{l}\text { Gastos no vinculados con intereses/activos } \\
\text { promedio }\end{array}$ & 145 & 4.3 & 6.4 & 0.7 & 41.9 \\
\hline $\begin{array}{l}\text { Activos líquidos/depósitos y financiamiento } \\
\text { de corto plazo }\end{array}$ & 145 & 24.5 & 16.0 & 4.3 & 117.6 \\
\hline Activos líquidos/total de depósitos y préstamos & 124 & 22.4 & 17.0 & 4.3 & 117.6 \\
\hline
\end{tabular}

Esta tabla presenta los estadísticos descriptivos de las variables bancarias. La muestra incluye 30 bancos centroamericanos y el periodo estudiado comprende los años 2008 a 2012.

a Balance a final de año, en miles de dólares. El resto de variables son ratios en porcentaje.

Fuente: cálculos del autor usando información de Bankscope.

seguridad sobre la vulnerabilidad del capital base. También se usa la razón entre los préstamos dudosos y el total de préstamos: un menor ratio corresponde a una mayor calidad en los activos.

La calidad de la administración es medida con dos ratios. El primero es entre gastos no vinculados con intereses y el total de ingresos, donde usualmente la mayor parte de los gastos corresponden a salarios. Es una medida de eficiencia, entre menor mejor. Similarmente, el segundo ratio es entre los gastos no vinculados a intereses y el promedio de los activos.

Los indicadores de utilidad son dos típicos ratios. Primero, el rendimiento de los activos (ROA), quizás el indicador más popular sobre desempeño operacional y eficiencia. Segundo, el rendimiento del patrimonio (ROE) sobre el capital accionario. Entre mayor sea esta cifra mejor, excepto en caso de elevados niveles de apalancamiento.

Finalmente, la liquidez bancaria es aproximada con el ratio activos líquidos sobre depósitos y financiamiento de corto plazo. Indica el porcentaje en que el financiamiento de corto plazo puede ser cubierto en caso de retiros súbitos. Un mayor porcentaje indica una mejor liquidez bancaria y menor vulnerabilidad a pánico bancario. Un segundo indicador es activos líquidos entre el total de depósitos y préstamos.

Así pues, se tienen dos indicadores para cada una de las categorías del sistema CAMUL, excepto capitalización. No es de sorprender la alta correlación que se presenta entre cada par (tabla 3); consecuentemente, en el análisis econométrico estas variables son incluidas evitando multicolinealidad, a la vez que permiten verificar la robustez de los resultados a diversos indicadores de los fundamentales bancarios. 
Tabla 3

Matriz de correlaciones

\begin{tabular}{|c|c|c|c|c|c|c|c|}
\hline & (1) & (2) & (3) & (4) & $(5)$ & (6) & (7) \\
\hline Activos totales (1) & 1.00 & & & & & & \\
\hline Depósitos totales (2) & 0.99 & 1.00 & & & & & \\
\hline Tasa de interés por depósitos (3) & -0.26 & -0.25 & 1.00 & & & & \\
\hline Tasa de crecimiento de los depósitos (4) & -0.27 & -0.25 & 0.61 & 1.00 & & & \\
\hline Tasa de crecimiento de los préstamos (5) & -0.20 & -0.17 & 0.15 & 0.54 & 1.00 & & \\
\hline Ratio de adecuación del capital (6) & -0.22 & -0.22 & 0.00 & -0.01 & -0.25 & 1.00 & \\
\hline Reservas para préstamos dudosos/total de préstamos dudosos (7) & -0.15 & -0.13 & -0.08 & 0.16 & 0.37 & -0.10 & 1.00 \\
\hline Préstamos dudosos/total de préstamos (8) & -0.01 & -0.02 & -0.07 & -0.16 & -0.38 & 0.69 & -0.37 \\
\hline ROA ingreso neto/activos totales promedio (9) & -0.07 & -0.05 & -0.10 & -0.11 & -0.12 & 0.76 & 0.05 \\
\hline ROE ingreso neto/acciones totales promedio (10) & 0.06 & 0.09 & -0.14 & -0.13 & 0.12 & 0.02 & 0.30 \\
\hline Gastos no vinculados con intereses/total de ingresos (11) & -0.08 & -0.09 & 0.20 & 0.35 & 0.18 & 0.09 & -0.08 \\
\hline Gastos no vinculados con intereses/activos promedio (12) & -0.14 & -0.14 & 0.10 & 0.05 & -0.14 & 0.86 & -0.14 \\
\hline Activos líquidos/depósitos y financiamiento de corto plazo (13) & -0.20 & -0.22 & 0.07 & 0.18 & -0.01 & 0.80 & -0.01 \\
\hline \multirow[t]{2}{*}{ Activos líquidos/total de depósitos y préstamos (14) } & -0.21 & -0.22 & 0.03 & 0.13 & -0.12 & 0.86 & -0.04 \\
\hline & (8) & (9) & $(10)$ & $(11)$ & (12) & (13) & (14) \\
\hline Préstamos dudosos/total de préstamos (8) & 1.00 & & & & & & \\
\hline ROA ingreso neto/activos totales promedio (9) & 0.44 & 1.00 & & & & & \\
\hline ROE ingreso neto/acciones totales promedio (10) & -0.26 & 0.55 & 1.00 & & & & \\
\hline Gastos no vinculados con intereses/total de ingresos (11) & 0.22 & -0.20 & -0.55 & 1.00 & & & \\
\hline Gastos no vinculados con intereses/activos promedio (12) & 0.69 & 0.77 & 0.01 & 0.30 & 1.00 & & \\
\hline Activos líquidos/depósitos y financiamiento de corto plazo (13) & 0.61 & 0.59 & -0.05 & 0.25 & 0.77 & 1.00 & \\
\hline Activos líquidos/total de depósitos y préstamos (14) & 0.65 & 0.65 & -0.03 & 0.23 & 0.80 & 0.99 & 1.00 \\
\hline
\end{tabular}

Esta tabla presenta los coeficientes de correlación de Pearson entre las principales variables bancarias. La muestra incluye 30 bancos centroamericanos y el periodo estudiado comprende los años 2008 a 2012.

Fuente: cálculos del autor usando información de Bankscope. 


\section{Modelo econométrico y resultados}

Es importante notar que existen problemas de endogeneidad entre las variables dependientes e independientes. Para poder controlar la doble causalidad, así como errores de especificación por no incluir todos los factores que afectan la tasa de interés y el crecimiento de los depósitos, es necesario utilizar variables instrumentales. Sin embargo, la carencia de datos imposibilita el hallazgo de instrumentos. Debido a estas dificultades, Goday et al. (2005) y Tovar-García (2014) recomiendan utilizar los estimadores DIF GMM o SYS GMM (Arellano y Bond, 1991; Blundell y Bond, 1998), métodos que todavía han sido poco empleados para contrastar la hipótesis de disciplina de mercado.

Dichos estimadores se basan en el método general de momentos (GMM) y utilizan un modelo dinámico para datos de panel, lo que permite controlar las características autorregresivas de las variables dependientes, es decir, se incluyen rezagos de la variable dependiente como regresor. El método DIF GMM estima una ecuación en primeras diferencias utilizando como variables instrumentales los rezagos de las variables independientes. Sin embargo, se encontró que dicha estimación puede estar sesgada y resultar en instrumentos débiles cuando las series son altamente persistentes, es decir, series que son altamente autorregresivas (Blundell, Bond y Windmeijer, 2001). En ese caso, la recomendación es utilizar el método SYS GMM, que estima una ecuación en niveles e incluye rezagos en primeras diferencias, y además rezagos en niveles de las variables independientes como instrumentos. No obstante, en el caso de muestras pequeñas el estimador GMM, eficiente en dos etapas, puede estar sesgado, por lo que Windmeijer (2005) sugiere una corrección a los errores estándar para obtener así una estimación más robusta. Posteriormente, Bun y Windmeijer (2010) subrayan las ventajas del método SYS GMM sobre el método DIF GMM, lo que ha llevado al primero a ser el método preferido en la mayor parte de las investigaciones aplicadas de los últimos años. Además, se recomienda su uso cuando el número de observaciones en el tiempo es mucho menor que el número de individuos en la muestra, como en el presente caso.

Así pues, SYS GMM en dos etapas es el principal método utilizado en esta investigación, cuyas estimaciones serán consistentes e insesgadas si no hay autocorrelación, particularmente de segundo orden, y si los instrumentos son válidos, para lo cual se emplea la prueba de Sargan. Sin embargo, cabe reconocer que el estimador SYS GMM puede padecer de instrumentos débiles cuando el tamaño de muestra es pequeño, con relativamente pocas observaciones temporales y de corte transversal (Bun y Windmeijer, 2010).

El modelo base para contrastar la hipótesis de disciplina de mercado está dado por la ecuación (1):

$$
\begin{aligned}
& \text { Dependiente }_{i t}=\beta_{1} \text { Dependiente }_{i t-1}+\text { CAMUL }_{i t-1}^{\prime} \alpha+\beta_{2} \text { TAMAN }^{\prime} O_{i t-1} \\
& +\beta_{3} \text { Crecimientoprestamos }_{i t-1}+\text { MACRO }_{i t}^{\prime} \beta+\phi_{1} \text { SEGURO } \\
& +\phi_{2} \text { PANAMA }_{i}+T_{t}^{\prime} \tau+u_{i t}
\end{aligned}
$$

Donde Dependiente incluye las variables tasa de interés por depósitos y tasa de crecimiento de los depósitos, para verificar la hipótesis de disciplina de mercado vía precio y cantidad, respectivamente.

CAMUL incluye los indicadores de capitalización, calidad de los activos, administración, utilidades y liquidez (presentados en la sección previa), evitando problemas de multicolinealidad y permitiendo verificar la robustez de los resultados a diferentes indicadores de los 
fundamentales bancarios. Nótese que estas variables son incluidas con un rezago debido a la demora en que la información es recibida y procesada por los depositantes (además, ayuda a controlar la endogeneidad).

La variable TAMAÑO es el logaritmo de los activos bancarios, un indicador típico de tamaño bancario, que permite controlar este efecto y la política implícita de demasiado grande para quebrar. No se tienen datos para poder incluir otras medidas del poder de mercado de cada banco, por lo que se supone que el tamaño bancario es también una variable que permite controlar dicho poder.

Es importante notar que el modelo incluye la variable Crecimientoprestamos (tasa de crecimiento de los préstamos) para controlar el efecto de la demanda interna de capital del banco (Ben-David et al., 2016; Tovar-García, 2014).

MACRO incluye variables macroeconómicas: tasa de crecimiento del PIB, inflación y la mediana de la tasa de interés por depósitos (la fuente es WDI) ${ }^{7}$; así, el modelo controla los efectos macroeconómicos sobre las variables dependientes para cada país. SEGURO es una variable dicotómica (dummy) que toma el valor de 1 si el país presenta un sistema de seguro de depósitos explícito y limitado, y el valor 0 en caso de tener un seguro de depósito implícito, como es el caso de Panamá y de Costa Rica $^{8}$. PANAMA es una variable dicotómica que toma valores de 1 para los bancos que operan en Panamá y 0 en caso contrario. Nótese que los bancos en Panamá son mayoría en la muestra, y con esta variable ficticia se puede controlar su efecto y las conclusiones generales para América Central ${ }^{9}$. Finalmente, $T$ es una variable dicotómica para los años de estudio, controlando condiciones del mercado que no pueden especificarse.

\section{Mecanismo basado en precio}

Cuando la variable dependiente es la tasa de interés por los depósitos, las hipótesis estadísticas indican una relación negativa con los fundamentales bancarios. Es decir, se espera una relación negativa con el ratio de adecuación del capital, reservas para préstamos dudosos/total de préstamos dudosos, ROA, ROE, activos líquidos/depósitos y financiamiento de corto plazo, y activos líquidos/total de depósitos y préstamos. Por el contrario, se espera una relación positiva con préstamos dudosos/total de préstamos, gastos no vinculados con intereses/total de ingresos, y gastos no vinculados con intereses/activos promedio.

Además, es de esperar una relación positiva entre la tasa de interés por los depósitos y la tasa de crecimiento de los préstamos, lo que es interpretado a favor de la hipótesis de demanda interna de capital (Ben-David et al., 2016). Se presentan varias regresiones incluyendo y excluyendo la tasa de crecimiento de los préstamos para evaluar su impacto en la tasa de interés y en la hipótesis de disciplina de mercado (el efecto de los fundamentales bancarios).

Los principales resultados se reportan en la tabla 4. El modelo dinámico se justifica adecuadamente; nótese que la variable dependiente como regresor presenta significancia estadística. Las

\footnotetext{
${ }^{7}$ En El Salvador se comenzó un proceso de dolarización en el año 2001; consecuentemente, se utiliza la tasa de interés de referencia de Estados Unidos (bill rate) como variable macroeconómica de control para este país.

${ }^{8}$ No se tienen indicadores sobre otras variables regulatorias o sobre la calidad institucional de cada país. Sin embargo, suponiendo que estos aspectos institucionales cambian poco en el tiempo, la estructura de los datos de panel permite controlar su posible impacto.

${ }^{9}$ Debido a problemas de multicolinealidad (principalmente con las variables macroeconómicas), no es posible incluir variables dicotómicas para otros países.
} 


\begin{tabular}{|c|c|c|c|c|c|c|c|c|}
\hline & \multirow{2}{*}{$\begin{array}{l}\text { Signo } \\
\text { esperado }\end{array}$} & \multicolumn{7}{|c|}{ Dependiente: tasa de interés por depósitos } \\
\hline & & $\begin{array}{l}\text { SYS GMM } \\
\text { (1) }\end{array}$ & $\begin{array}{l}\text { SYS GMM } \\
\text { (2) }\end{array}$ & $\begin{array}{l}\text { SYS GMM } \\
\text { (3) }\end{array}$ & $\begin{array}{l}\text { SYS GMM } \\
\text { (4) }\end{array}$ & $\begin{array}{l}\text { DIF GMM } \\
\text { (5) }\end{array}$ & $\begin{array}{l}\text { Efectos fijos } \\
\text { (6) }\end{array}$ & $\begin{array}{l}\text { Efectos } \\
\text { flexibles (7) }\end{array}$ \\
\hline Dependiente rezagada & & $0.67^{* * *}$ & $0.63^{* * * *}$ & $0.67^{* * * * *}$ & $0.65^{* * *}$ & $0.67^{* * * *}$ & & \\
\hline Ratio de adecuación del capital & - & -0.01 & 0.02 & -0.04 & -0.002 & -0.03 & -0.04 & -0.02 \\
\hline Reservas para préstamos dudosos/total de préstamos dudosos & - & -0.001 & & $-0.001^{*}$ & & $-0.001^{*}$ & -0.0003 & -0.0003 \\
\hline ROA & - & $-0.35^{* * *}$ & & $-0.40^{* * *}$ & & $-0.39^{* *}$ & -0.31 & -0.20 \\
\hline Gastos no vinculados con intereses/total de ingresos & + & 0.01 & & 0.01 & & 0.01 & 0.004 & 0.01 \\
\hline Activos líquidos/depósitos y financiamiento de corto plazo & - & -0.01 & & -0.02 & & -0.01 & 0.01 & 0.001 \\
\hline Préstamos dudosos/total de préstamos & + & & 0.03 & & 0.04 & & & \\
\hline ROE & - & & $-0.03^{*}$ & & $-0.03^{*}$ & & & \\
\hline Gastos no vinculados con intereses/activos promedio & + & & $-0.18^{* *}$ & & -0.14 & & & \\
\hline Activos líquidos/total de depósitos y préstamos & - & & -0.03 & & -0.01 & & & \\
\hline Logaritmo del total de activos (tamaño bancario) & & $0.17^{*}$ & $0.25^{* * *}$ & $0.27^{* * *}$ & $0.30^{* * * *}$ & 0.20 & -1.11 & $-0.57^{* * * *}$ \\
\hline Tasa de crecimiento de los préstamos & + & & & $-0.01^{*}$ & $-0.01^{* * * *}$ & $-0.01^{*}$ & -0.01 & -0.01 \\
\hline Tasa de crecimiento del PIB & & $-0.19^{* *}$ & $-0.18^{* *}$ & $-0.16^{* *}$ & -0.08 & $-0.15^{* *}$ & -0.04 & 0.03 \\
\hline Inflación & & -0.05 & $-0.09^{* *}$ & -0.05 & $-0.10^{* * *}$ & $-0.05^{*}$ & -0.07 & -0.12 \\
\hline Tasa de interés nacional por depósitos (mediana) & & 0.17 & 0.18 & 0.21 & 0.18 & 0.19 & 0.03 & $0.28^{* * * *}$ \\
\hline Seguro de depósito (dummy) & & -0.03 & -2.05 & -1.78 & -2.35 & & & -0.29 \\
\hline Panamá (dummy) & & -1.03 & 0.18 & -1.63 & -0.97 & & & 0.38 \\
\hline Año 2010 (dummy) & & 0.11 & -0.02 & -0.01 & -0.58 & -0.08 & $-0.86^{*}$ & $-0.98^{* *}$ \\
\hline Año 2011 (dummy) & & 0.76 & 0.70 & 0.63 & 0.01 & 0.51 & -0.83 & -0.95 \\
\hline Año 2012 (dummy) & & $0.99^{*}$ & $0.87^{*}$ & $0.95^{*}$ & 0.35 & 0.85 & -0.73 & -0.96 \\
\hline Periodo & & 2008-2012 & & & & & & \\
\hline Observaciones & & 95 & 85 & 95 & 85 & 67 & 95 & 95 \\
\hline $\mathrm{N} \times \mathrm{T}$ & & $27 \times 4$ & $23 \times 4$ & $27 \times 4$ & $23 \times 4$ & $24 \times 3$ & $27 \times 4$ & $27 \times 4$ \\
\hline Prueba de Sargan & & $3.37(0.76)$ & $9.69(0.14)$ & $5.68(0.46)$ & $8.65(0.19)$ & $6.11(0.29)$ & & \\
\hline Prueba de correlación de primer orden (significancia) & & -2.05 & -2.08 & -2.18 & -2.22 & -2.37 & & \\
\hline & & $(0.04)$ & $(0.04)$ & $(0.03)$ & $(0.03)$ & $(0.02)$ & & \\
\hline Prueba de correlación de segundo orden (significancia) & & -0.10 & -0.35 & -0.32 & -0.66 & -0.41 & & \\
\hline & & $(0.92)$ & $(0.73)$ & $(0.75)$ & $(0.51)$ & $(0.68)$ & & \\
\hline
\end{tabular}

Esta tabla presenta los resultados de las regresiones usando el estimador SYS GMM (Blundell y Bond, 1998), en dos etapas. Asimismo, se presentan resultados con el estimador DIF GMM (Arellano y Bond, 1991) y regresiones con efectos fijos y flexibles. Las variables independientes (en las filas) entran con un rezago y la variable dependiente es la tasa de interés por depósitos (léase las regresiones verticalmente). La muestra incluye observaciones anuales de 2008 a 2012 de 30 bancos comerciales centroamericanos. Los datos bancarios son tomados de Bankscope, y las variables macroeconómicas, del Banco Mundial (WDI).

* Significancia estadística al $10 \%$.

** Significancia estadística al $5 \%$.

*** Significancia estadística al $1 \%$. 
estimaciones con el SYS GMM (columnas 1 a 4) no presentan problemas de autocorrelación de segundo orden y los instrumentos son válidos de acuerdo con la prueba de Sargan.

En general, la evidencia a favor de la hipótesis de disciplina de mercado vía precio es débil. Solamente las variables rendimiento de los activos y del patrimonio (ROA y ROE) presentan significancia estadística y el signo esperado, es decir, los bancos con rendimientos más altos pagan menos por los depósitos. El resto de los fundamentales bancarios no muestra significancia estadística o su efecto carece de robustez al no presentar significancia en la mayoría de las regresiones.

Se podría argumentar que los depositantes monitorean principalmente la rentabilidad de su banco como indicador clave para tomar decisiones sobre el precio al cual colocar sus depósitos, pero también cabe pensar que es la baja tasa de interés por los depósitos la que facilita a estos bancos obtener mejores rendimientos. Al no encontrar la relación esperada con otros fundamentales bancarios, los resultados sugieren una débil presencia de disciplina de mercado vía precio.

Nótese que las dos primeras columnas excluyen la tasa de crecimiento de los préstamos como variable independiente; esta se incluye en las regresiones de las columnas 3 y 4 , por lo que el efecto de los fundamentales bancarios sobre la tasa de interés de los depósitos no se ve afectado. Es decir, solamente ROA y ROE siguen presentando el efecto predicho por la hipótesis de disciplina de mercado. Sorprendentemente, la tasa de crecimiento de los préstamos presenta significancia estadística con el signo contrario al esperado, contradiciendo la hipótesis de demanda interna de capital del banco. En otras palabras, los bancos que más prestan pagan menos por los depósitos, no necesitan ofrecer tasas de interés más altas para atraer depósitos con los cuales enfrentar la demanda de préstamos. Esto implica que la actividad crediticia del banco depende de otros fondos y no de los depósitos. No obstante, lo anterior requiere pruebas más sofisticadas y enfocadas, lo que sobrepasa los límites de esta investigación ${ }^{10}$. Aquí esta variable es incluida principalmente para controlar el efecto de los fundamentales bancarios, que no presentan cambios relevantes.

El tamaño bancario presenta un efecto positivo y robusto sobre la tasa de interés por los depósitos (es estadísticamente significativo en las cuatro regresiones). En otras palabras, los bancos más grandes pagan tasas de interés más altas por los depósitos, lo que implica la carencia de una política implícita de demasiado grande para quebrar.

De entre las variables macroeconómicas, la tasa de crecimiento del PIB y la inflación presentan algunos coeficientes significativos y negativos. En particular el crecimiento del PIB parece impactar negativamente en la tasa de interés por depósitos. Las variables dicotómicas para los años de estudio no presentan efectos estadísticamente significativos en la mayoría de las regresiones, a excepción del año 2012, con un efecto positivo, sugiriendo la salida de la crisis global.

La variable dicotómica para el seguro de depósito no muestra significancia estadística, es decir, tener un seguro explícito o un seguro implícito (como en Costa Rica y Panamá) no afecta las tasas de interés pagadas por los depósitos.

\section{Mecanismo basado en cantidad}

Cuando la variable dependiente es la tasa de crecimiento de los depósitos, las hipótesis estadísticas indican una relación positiva con los fundamentales bancarios. Es decir, se espera una relación positiva con el ratio de adecuación del capital, reservas para préstamos dudosos/total

\footnotetext{
$\overline{10}$ Entre las distintas pruebas que se hicieron, cabe mencionar que la variable crecimiento de los depósitos al ser introducida sin rezago, presenta un efecto positivo y significativo sólo en una de las dos regresiones.
} 
de préstamos dudosos, ROA, ROE, activos líquidos/depósitos y financiamiento de corto plazo, y activos líquidos/total de depósitos y préstamos. Por el contrario, se espera una relación negativa con préstamos dudosos/total de préstamos, gastos no vinculados con intereses/total de ingresos, y gastos no vinculados con intereses/activos promedio.

Asimismo, es de esperar una relación positiva entre la tasa de crecimiento de los depósitos y la tasa de crecimiento de los préstamos, lo que es interpretado a favor de la hipótesis de demanda interna de capital.

Los principales resultados se reportan en la tabla 5. Las estimaciones con el SYS GMM (columnas 1 a 4) no presentan problemas de autocorrelación de segundo orden y los instrumentos son válidos de acuerdo con la prueba de Sargan.

A través del mecanismo basado en cantidad, la evidencia es contraria a la hipótesis de disciplina de mercado. La capitalización bancaria tiene un efecto negativo, significativo y robusto sobre la tasa de crecimiento de los depósitos, es decir, los bancos mejor capitalizados obtienen menos depósitos.

Además, los indicadores de la calidad de los activos presentan significancia estadística y el signo contrario al esperado. Es decir, los bancos con menor calidad en sus activos obtienen más depósitos. Nótese que el resultado es robusto; las variables reservas para préstamos dudosos sobre total de préstamos dudosos, y préstamos dudosos sobre total de préstamos, presentan evidencia en contra de la hipótesis de disciplina de mercado en las cuatro regresiones; las dos últimas (columnas 3 y 4) incluyen la tasa de crecimiento de los préstamos como variable independiente, misma que no presenta significancia estadística, ni modifica el efecto de los fundamentales bancarios sobre la tasa de crecimiento de los depósitos. Consecuentemente, tampoco hay evidencia a favor de la hipótesis de demanda interna de capital del banco, a diferencia del caso estadounidense, donde esta determina las tasas de interés y la cantidad de depósitos (Ben-David et al., 2016).

El resto de los fundamentales bancarios no presentan efectos estadísticamente significativos, ni robustos. El tamaño bancario, al igual que en el mecanismo basado en precio, presenta significancia estadística y robustez, y su efecto sobre la tasa de crecimiento de los depósitos es negativo, es decir, los bancos más grandes atraen menos depósitos, soportando el hallazgo previo en contra de una política implícita de demasiado grande para quebrar. Así pues, la evidencia contradice la hipótesis de disciplina de mercado: los depositantes no reaccionan de la manera esperada a los fundamentales bancarios y tampoco al tamaño del banco. No obstante, este último resultado debe ser tratado con cautela, porque la muestra incluye principalmente bancos grandes en sus respectivos países.

Los factores macroeconómicos y la tendencia en el tiempo capturada en las variables dicotómicas para los años de estudio no presentan efectos estadísticamente significativos. En cambio, la variable dicotómica para los bancos que operan en Panamá presenta un efecto positivo y significativo, es decir, estos bancos presentan tasas de crecimiento de los depósitos mayores al resto de los bancos centroamericanos.

A diferencia del mecanismo basado en precio, la variable dicotómica para seguro de depósito presenta significancia estadística y un efecto positivo, es decir, las tasas de crecimiento de los depósitos son mayores en los países que tienen un esquema de seguro explícito y limitado, implicando que el seguro de depósito da mayor confianza a los depositantes.

Cabe señalar que la motivación en la investigación de Mayorga Martínez y Muñoz Salas (2002) era la discusión sobre la inclusión de un seguro de depósito explícito y limitado para Costa Rica. Por un lado, el seguro de depósito da certeza sobre la intervención del estado en caso de quiebra bancaria y evita el pánico bancario. Por otro lado, existe evidencia empírica que sugiere que los seguros de depósitos debilitan la disciplina de mercado (Demirgüç-Kunt y Huizinga, 2004; 
Tabla 5

Disciplina de mercado: Mecanismo basado en cantidad

\begin{tabular}{|c|c|c|c|c|c|c|c|c|}
\hline \multirow[t]{2}{*}{ Signo esperado } & \multicolumn{7}{|c|}{ Dependiente: tasa de crecimiento de los depósitos } & \\
\hline & $\begin{array}{l}\text { SYS GMM } \\
\text { (1) }\end{array}$ & $\begin{array}{l}\text { SYS GMM } \\
\text { (2) }\end{array}$ & $\begin{array}{l}\text { SYS GMM } \\
\text { (3) }\end{array}$ & $\begin{array}{l}\text { SYS GMM } \\
\text { (4) }\end{array}$ & $\begin{array}{l}\text { DIF GMM } \\
\text { (5) }\end{array}$ & $\begin{array}{l}\text { Efectos fijos } \\
\text { (6) }\end{array}$ & $\begin{array}{l}\text { Efectos } \\
\text { flexibles (7) }\end{array}$ & \\
\hline Dependiente rezagada & & 0.17 & $0.15^{*}$ & 0.19 & $0.17^{*}$ & 0.19 & & \\
\hline Ratio de adecuación del capital & + & $-1.40^{\text {*** }}$ & $-2.08^{* * * *}$ & $-1.58^{* * * *}$ & $-2.20^{* * * *}$ & $-1.60^{* * *}$ & -1.04 & 0.02 \\
\hline Reservas para préstamos dudosos/total de préstamos dudosos & + & $-0.03^{* *}$ & & $-0.02^{* *}$ & & $-0.02^{* *}$ & -0.02 & -0.004 \\
\hline ROA & + & 2.82 & & $2.98^{*}$ & & $3.06^{*}$ & 2.41 & 3.20 \\
\hline Gastos no vinculados con intereses/total de ingresos & - & -0.29 & & -0.25 & & -0.25 & 0.07 & $0.39^{* * * *}$ \\
\hline Activos líquidos/depósitos y financiamiento de corto plazo & + & -0.30 & & -0.32 & & -0.35 & 0.13 & $-0.39^{* *}$ \\
\hline Préstamos dudosos/total de préstamos & - & & $3.76^{* * *}$ & & $3.86^{* * * *}$ & & & \\
\hline ROE & + & & -0.32 & & -0.28 & & & \\
\hline Gastos no vinculados con intereses/activos promedio & - & & 0.69 & & 0.64 & & & \\
\hline Activos líquidos/total de depósitos y préstamos & + & & -0.24 & & -0.24 & & & \\
\hline Logaritmo del total de activos (tamaño bancario) & & $-57.2^{* * * *}$ & $-48.9^{* * *}$ & $-54.3^{* * * *}$ & $-46.4^{* * *}$ & $-52.8^{* * * *}$ & $-29.55^{* *}$ & $-5.57^{* * * *}$ \\
\hline Tasa de crecimiento de los préstamos & + & & & -0.12 & -0.05 & -0.13 & -0.05 & $0.23^{* * * *}$ \\
\hline Tasa de crecimiento del PIB & & 0.20 & -0.43 & 0.43 & -0.67 & 0.18 & -3.61 & -0.80 \\
\hline Inflación & & 1.19 & 1.17 & 1.10 & 1.28 & 0.86 & -0.005 & -1.29 \\
\hline Tasa de interés nacional por depósitos (mediana) & & -0.86 & -1.52 & -0.43 & -2.41 & -0.63 & -5.48 & 0.35 \\
\hline Seguro de depósito (dummy) & & $2,148^{* * *}$ & $1,395^{* * *}$ & $2,113^{* * *}$ & $1,312^{\text {*** }}$ & & & -10.67 \\
\hline Panamá (dummy) & & $721^{* * * *}$ & $619^{* * * *}$ & $674^{* * * *}$ & $593^{* * * *}$ & & & 3.50 \\
\hline Año 2010 (dummy) & & 1.06 & 0.98 & -0.85 & 0.60 & 0.26 & 11.49 & 6.81 \\
\hline Año 2011 (dummy) & & -1.04 & 0.33 & -3.27 & -0.27 & -0.93 & 16.22 & 9.22 \\
\hline Año 2012 (dummy) & & 6.72 & 9.67 & 5.24 & 9.26 & 7.05 & 16.34 & 4.50 \\
\hline Periodo & & $2008-2012$ & & & & & & \\
\hline Observaciones & & 95 & 85 & 95 & 85 & 67 & 95 & 95 \\
\hline $\mathrm{N} \times \mathrm{T}$ & & $27 \times 4$ & $23 \times 4$ & $27 \times 4$ & $23 \times 4$ & $24 \times 3$ & $27 \times 4$ & $27 \times 4$ \\
\hline Prueba de Sargan (significancia) & & $5.86(0.43)$ & $6.02(0.42)$ & $6.17(0.40)$ & $5.76(0.45)$ & $6.01(0.30)$ & & \\
\hline Prueba de correlación de primer orden (significancia) & & -1.78 & -1.58 & -1.84 & -1.69 & -1.98 & & \\
\hline & & $(0.07)$ & $(0.11)$ & $(0.06)$ & $(0.09)$ & $(0.05)$ & & \\
\hline Prueba de correlación de segundo orden (significancia) & & -0.58 & -0.07 & -0.56 & -0.06 & -0.56 & & \\
\hline & & $(0.56)$ & $(0.94)$ & $(0.57)$ & $(0.95)$ & $(0.57)$ & & \\
\hline
\end{tabular}

Esta tabla presenta los resultados de las regresiones usando el estimador SYS GMM (Blundell y Bond, 1998), en dos etapas. Asimismo, se presentan resultados con el estimador DIF GMM (Arellano y Bond, 1991) y regresiones con efectos fijos y flexibles. Las variables independientes (en las filas) entran con un rezago y la variable dependiente es la tasa de crecimiento de los depósitos (léase las regresiones verticalmente). La muestra incluye observaciones anuales de 2008 a 2012 de 30 bancos comerciales centroamericanos. Los datos bancarios son tomados de Bankscope, y las variables macroeconómicas, del Banco Mundial (WDI).

* Significancia estadística al $10 \%$.

* Significancia estadística al 5\%.

**** Significancia estadística al $1 \%$. 
Sulamita Estrada, 2013). No obstante, los resultados aquí presentados sugieren débil presencia de disciplina de mercado vía precio, y su ausencia vía cantidad, para el periodo 2008-2012. Asimismo, Mayorga Martínez y Muñoz Salas (2002) reportan débil presencia de disciplina de mercado vía cantidad para el periodo 1995-2001. Consecuentemente, los argumentos en contra de la inclusión del seguro de depósito por afectar negativamente la disciplina de mercado carecen de sentido, ya que ni siquiera hay evidencia de su presencia.

En suma, no hay evidencia de disciplina de mercado en el sistema bancario centroamericano, y estos resultados coinciden en buena medida con los presentados por Mayorga Martínez y Muñoz Salas (2002) para el caso costarricense.

Cabe señalar que las regresiones también fueron estimadas usando errores estándar robustos conforme a Windmeijer (2005). Como es de esperar, los coeficientes enfrentan intervalos de aceptación de la hipótesis nula más amplios, reduciendo así, aún más, la posibilidad de alcanzar significancia estadística. Como medidas adicionales para verificar robustez se realizaron regresiones con diferentes combinaciones de los indicadores CAMUL y con una transformación logarítmica de las principales variables dependientes e independientes; los resultados son muy similares, pero usando logaritmos las regresiones pocas veces pasan la prueba de Sargan (estos resultados no son presentados en tablas). También se realizaron regresiones con el estimador DIF GMM y con efectos fijos y flexibles (véase columnas 5, 6 y 7 en las tablas 4 y 5). El estimador DIF GMM arroja resultados muy similares al SYS GMM. Las regresiones con efectos fijos y flexibles, aunque sabemos que están sesgadas por no controlar adecuadamente endogeneidad, presentan resultados ligeramente distintos al SYS GMM, pero coinciden al no presentar evidencia a favor de la hipótesis de disciplina de mercado.

Además, dado que la muestra está dominada por bancos que operan en Panamá, se realizaron los mismos ejercicios econométricos únicamente para estos bancos (no se presenta en tablas para ahorrar espacio). Los resultados obtenidos son cualitativamente los mismos, previsiblemente, porque la variable dicotómica PANAMA no presenta significancia estadística en las regresiones del mecanismo de disciplina basado en precio, y aunque sí presenta significancia estadística en el mecanismo basado en cantidad, no es más que el reflejo del mejor comportamiento macroeconómico panameño.

\section{Conclusión}

El sistema bancario es esencial para el adecuado desempeño de la economía. No obstante, durante las últimas décadas muchos de los problemas en el sector real tuvieron origen en el sector financiero. Hasta ahora no ha sido posible prever y prevenir las crisis bancarias, responsabilidad que suele atribuirse a los supervisores bancarios. Sin embargo, es importante señalar que los partícipes del mercado también tienen un papel importante en el monitoreo del riesgo bancario.

De acuerdo con el tercer pilar de Basilea III, una política de revelación de la información bancaria es importante para que los bancos enfrenten disciplina de mercado, donde los agentes económicos tomen decisiones que castiguen a los bancos por su conducta riesgosa. En el caso del mercado de depósitos, es de esperar que los bancos más riesgosos paguen tasas de interés más altas y atraigan menos fondos. Estas hipótesis han sido ampliamente verificadas en la literatura empírica, con evidencia a favor de la hipótesis de disciplina de mercado principalmente en países desarrollados, respaldando así las recomendaciones de Basilea (Berger y Turk-Ariss, 2014). La evidencia en países latinoamericanos es mixta, y hallazgos recientes en México sugieren una débil presencia de disciplina de mercado (Tovar-García, 2014). 
Además, los resultados empíricos previos pueden estar sesgados porque no controlan el efecto de la demanda interna de capital del banco, tal como sugieren hallazgos recientes para el caso estadounidense, donde al controlar dicho efecto no se observa que los bancos más riesgosos paguen tasas de interés más altas. Son los bancos que enfrentan un crecimiento en los préstamos los que pagan tasas más altas por los depósitos (Ben-David et al., 2016).

En esta investigación se verificó la hipótesis de disciplina de mercado en el sistema bancario centroamericano, la cual había sido contrastada solo para el caso costarricense, donde los resultados débilmente respaldan que los bancos más riesgosos atraen menos depósitos (Mayorga Martínez y Muñoz Salas, 2002).

Aquí se empleó una muestra de 30 bancos con presencia en Costa Rica, El Salvador, Guatemala, Honduras, Nicaragua y Panamá, y se estudió el periodo 2008-2012 en el marco de la crisis financiera global. Con base en el estimador SYS GMM (Blundell y Bond, 1998) se encontró débil evidencia de que los bancos más riesgosos pagan tasas de interés más altas. En específico, solo se encontró que los bancos con mejores rendimientos sobre los activos y el patrimonio (altos niveles de ROA y ROE) pagan menores tasas de interés por los depósitos. Consecuentemente, se puede decir que en el caso centroamericano no hay evidencia de disciplina de mercado.

Es importante señalar que el modelo econométrico básico corrige la doble causalidad, a diferencia de los modelos simples con efectos fijos o flexibles comúnmente empleados en la literatura previa, y que los principales hallazgos son robustos a diferentes indicadores de riesgo bancario (fundamentales bancarios), al efecto de la demanda interna de capital del banco y a otros métodos de estimación. Por consiguiente, la principal tarea de los encargados de la política bancaria es restaurar la disciplina de mercado, conforme a las sugerencias del Comité de Basilea, porque a excepción de Honduras, el resto de las naciones centroamericanas solo han presentado documentos preliminares con respecto a la implementación del tercer pilar (Financial Stability Institute BIS, 2013) y en el índice de monitoreo privado elaborado por Barth, Caprio y Levine (2013) están clasificadas en los lugares más bajos y con tendencias negativas.

La presente investigación presenta limitaciones derivadas de la disponibilidad de datos bancarios, lo que también ejemplifica la ausencia general de revelación de información bancaria en Centroamérica. La muestra aquí utilizada está dominada por bancos que operan en Panamá, y aunque se usan datos de panel y los resultados son robustos a distintos métodos de estimación, cabe ser cautos con generalizaciones. Futuras investigaciones deben mejorar el tamaño de muestra y extender el periodo de análisis.

\section{Referencias}

Arellano, M. y Bond, S. (1991). Some tests of specification for panel data: Monte Carlo evidence and an application to employment equations. The Review of Economic Studies, 58(2), 277-297. http://restud.oxfordjournals.org/ content/58/2/277.short

Ayadi, R. (2013). On the role of the Basel Committee, the Basel rules, and banks' incentives. En G. Caprio (Ed.), Handbook of Safeguarding Global Financial Stability (pp. 403-417). Elsevier Inc. http://dx.doi.org/10.1016/ B978-0-12-397875-2.00127-6

Barth, J. R., Caprio, G. y Levine, R. (2013). Bank regulation and supervision in 180 countries from 1999 to 2011. Journal of Financial Economic Policy, 5(2), 111-219. http://dx.doi.org/10.1108/17576381311329661

Basel Committee on Banking Supervision (2006). International Convergence of Capital Measurement and Capital Standards: A Revised Framework — Comprehensive Version. Disponible en: http://www.bis.org/publ/bcbs128.htm

Basel Committee on Banking Supervision (2011). Basel III: A global regulatory framework for more resilient banks and banking systems — revised version. Disponible en: http://www.bis.org/publ/bcbs189.htm

Basel Committee on Banking Supervision (2014). Review of the Pillar 3 disclosure requirements. Bank for International Settlements 2014. Disponible en: http://www.bis.org/publ/bcbs286.pdf 
Ben-David I, Palvia A, Spatt C (2016). Banks' internal capital markets and deposit rates. Journal of Financial and Quantitative Analysis (JFQA). Disponible en: https://papers.ssrn.com/sol3/papers.cfm?abstract_id=2646840\#

Berger AN, Turk-Ariss R (2014). Do depositors discipline banks and did government actions during the recent crisis reduce this discipline? An international perspective. Disponible en: http://www.mooreschool. sc.edu/UserFiles/moore/Documents/Finance/Berger/DoDepositorsDisciplineBanks.pdf

Blundell, R. y Bond, S. (1998). Initial conditions and moment restrictions in dynamic panel data models. Journal of Econometrics, 87(1), 115-143. http://dx.doi.org/10.1016/S0304-4076(98)00009-8

Blundell, R., Bond, S. y Windmeijer, F. (2001). Estimation in dynamic panel data models: Improving on the performance of the standard GMM estimator. Advances in Econometrics, 15, 53-91. http://dx.doi.org/10.1016/ S0731-9053(00)15003-0

Bun, M. J. G. y Windmeijer, F. (2010). The weak instrument problem of the system GMM estimator in dynamic panel data models. The Econometrics Journal, 13(1), 95-126. http://dx.doi.org/10.1111/j.1368-423X.2009.00299.x

Cubillas, E., Fonseca, A. R. y González, F. (2012). Banking crises and market discipline: International evidence. Journal of Banking \& Finance, 36(8), 2285-2298. http://dx.doi.org/10.1016/j.jbankfin.2012.04.011

Demirgüç-Kunt, A. y Huizinga, H. (2004). Market discipline and deposit insurance. Journal of Monetary Economics, 51(2), 375-399. http://dx.doi.org/10.1016/j.jmoneco.2003.04.001

Financial Stability Institute BIS. (2013). FSI Survey Basel II, 2.5 and III implementation. Disponible en: http://www.bis.org/index.htm

Flannery, M. J. (2001). The faces of market discipline. Journal of Financial Services Research, 20(2-3), 107-119. Disponible en: http://link.springer.com/article/10.1023/A:1012455806431

Goday, V., Gruss, B. y Ponce, J. (2005). Depositors' discipline in Uruguayan banks. Revista de Economía, 12(2), 168-204. Disponible en: http://dialnet.unirioja.es/descarga/articulo/3184214.pdf

Hasan, I., Jackowicz, K., Kowalewski, O. y Kozłowski, Ł. (2013). Market discipline during crisis: Evidence from bank depositors in transition countries. Journal of Banking \& Finance, 37(12), 5436-5451. http://dx.doi.org/10.1016/j.jbankfin.2013.06.007

Karas, A., Pyle, W. y Schoors, K. (2010). How do Russian depositors discipline their banks? Evidence of a backward bending deposit supply function. Oxford Economic Papers, 62(1), 36-61. http://dx.doi.org/10.1093/oep/gpp006

Márquez, J. (2011). El seguro de depósito y su incidencia en la disciplina de mercado en Colombia. Coyuntura Económica, 41(2), 87-117. Disponible en: http://www.fedesarrollo.org.co/wp-content/uploads/2012/08/C.-E. -Diciembre-2011El-seguro-de-dep\%25C3\%25B3sito-y-su-incidencia-pp.-87-117.pdf

Martinez-Peria, M. S. y Schmukler, S. L. (2001). Do depositors punish banks for bad behavior? Market discipline, deposit insurance, and banking crises. The Journal of Finance, 56(3), 1029-1051. Disponible en: http://onlinelibrary.wiley.com/doi/10.1111/0022-1082.00354/Abstract

Martínez Castillo, C. A. (2007). Basilea II, retos y oportunidades. Hacia una mayor armonización de la regulación y supervisión financiera en el siglo XXI. Gestión y Política Pública, 16(2), 465-510. http://www.gestionypoliticapublica. cide.edu/num_anteriores/Vol.XVI_No.II_2dosem/Martinez_Castillo.pdf

Mayorga Martínez, M. y Muñoz Salas, E. (2002). ¿Existe disciplina de mercado en el sistema bancario costarricense? Economía y Sociedad, (20), 61-100. http://www.revistas.una.ac.cr/index.php/economia/article/view/1174

Muñoz, J. F., Cabeza, J. L. y Guerra, W. (2013). Disciplina de mercado en la banca venezolana. Período $2004-2011$. Actualidad Contable FACES, 16(27), 87-101. Disponible en: http://www.saber.ula.ve/handle/123456789/37944

Park, S. (1995). Market discipline by depositors: Evidence from reduced-form equations. The Quarterly Review of Economics and Finance, 35, 497-514. http://dx.doi.org/10.1016/1062-9769(95)90051-9

Park, S. y Peristiani, S. (1998). Market discipline by thrift depositors. Journal of Money, Credit and Banking, 30(3), 347-364. Disponible en: http://www.jstor.org/stable/10.2307/2601105

Ríos B (2012). Ranking bancos: El oxígeno de la banca global. Mercados \& Tendencias, 32-59. Disponible en: http://revistamyt.com/wp-content/uploads/2012/07/ranking-de-bancos-2012.pdf

Sarkar, A. y Bhole, L. M. (2009). Bank depositors' role as a disciplinary force in Indian banking: A dynamic panel approach. En R. P. Padran (Ed.), Forecasting Financial Markets in India (pp. 30-39). India: Allied Publishers Private Limited. Disponible en: https://books.google.com.mx/books?id=D_8eoSMaD8cC\& $\mathrm{pg}=\mathrm{PA} 118 \& \mathrm{lpg}=\mathrm{PA} 118 \& \mathrm{dq}=\% 22$ Forecasting+Financial+Markets+in+India $\% 22 \&$ source=bl\&ots=nrP7Awq2Dr\& sig=QfPAqCln3hRDQiETcTR3XapOEdA\&hl=en\&sa=X\&ved= 0ahUKEwj49OO80tTSAhUX5GMKHbxPCQMQ6AEIIzAC\#v=onepage\&q=Sarkar\&f=fal

Sulamita Estrada, Y. (2013). El fondo de seguro de depósito en la estabilidad financiera en Centroamérica, República Dominicana y Panamá. Notas Económicas Regionales, (64), 1-11. http://www.secmca.org/ NOTAS_ECONOMICAS/articulo64MAY2013.pdf 
Tovar-García, E. D. (2012). Market discipline in Mexican banks: Evidence from the asset side. Cuadernos de Economía: Spanish Journal of Economics and Finance, 35(99), 172-181. http://dx.doi.org/10.1016/S0210-0266(12)70033-X

Tovar-García, E. D. (2014). Market discipline: A review of the Mexican deposit market. Latin American Economic Review, 23(6), 1-33. http://dx.doi.org/10.1007/s40503-014-0006-2

Tovar-García, E. D. (2015). Market discipline through subordinated debt in Mexican banks. Revista de Economia Aplicada, 23(68), 61-80. http://www.revecap.com/revista/aceptados/Tovar.pdf

Tovar-García, E. D. (2016a). Exposure to interbank market and risk-taking by Mexican banks. Cuadernos de Economía: Spanish Journal of Economics and Finance, 39(111), 157-174. http://dx.doi.org/10.1016/j.cesjef.2015.11.002

Tovar-García, E. D. (2016b). Who can better monitor a bank than another bank? Mechanisms of discipline in the Mexican interbank market. Revista de Métodos Cuantitativos para la Economía y la Empresa, 21(1), 205-229. Disponible en: http://www.upo.es/RevMetCuant/art.php?id=123

Tovar-García, E. D. y Kozubekova, R. (2016). The Third Pillar of the Basel Accord: Evidence of borrower discipline in the Kyrgyz banking system. Journal of Eurasian Studies, 7(2), 195-204. http://dx.doi.org/10.1016/j.euras.2016.02.002

Windmeijer, F. (2005). A finite sample correction for the variance of linear efficient two-step GMM estimators. Journal of Econometrics, (126), 25-51. http://dx.doi.org/10.1016/j.jeconom.2004.02.005

Wu, Y. y Bowe, M. (2012). Information disclosure and depositor discipline in the Chinese banking sector. Journal of International Financial Markets, Institutions and Money, 22(4), 855-878. http://dx.doi.org/10.1016/j.intfin.2012.05.004 